\title{
STABILITY OF QUADRATIC PROJECTION METHODS
}

\author{
LyONELL BOULTON AND MichaEL STRAUSS
}

Abstract. We discuss stability properties of the method studied recently in [7] and [2], for computing eigenvalues in gaps of the essential spectrum of self-adjoint operators.

Mathematics subject classification (2000): 47B36, 47B39, 81-08.

Key words and phrases: Non-variational projection methods, spectral pollution, numerical approximation of the spectrum.

\section{REFERENCES}

[1] L. Boulton, Limiting set of second order spectrum, to appear Math. Comp., 75 (2006) 1367-1382.

[2] L. Boulton, Non-variational approximation of discrete eigenvalues of self-adjoint operators, IMA J. Numer. Anal., 27 (2007) 102-121.

[3] M. Dauge, M. Suri, Numerical approximation of the spectra of non-compact operators arising in buckling problems, J. Numer. Math., 10 (2002), 193-219.

[4] E. B. Davies, Spectral enclosures and complex resonances for general self-adjoint operators, LMS J. Comput. Math., 1 (1998) 42-74.

[5] N. J. Higham, F. Tisseur, Structured pseudospectra for polynomial eigenvalue problems with applications, SIAM J. Matrix Anal. Appl., 23 (2001) 187-208.

[6] P. Lancaster, P. Psarrakos, On the pseudospectra of matrix polynomial, SIAM J. Matrix Anal. Appl., 27 (2005) 115-129.

[7] M. Levitin, E. Shargorodsky, Spectral pollution and second order relative spectra for self-adjoint operators, IMA J. Numer. Anal., 24 (2004) 393-416.

[8] E.B. Davies, M. Plum, Spectral pollution, IMA J. Numer. Anal., 24 (2004) 417-438.

[9] J. Rapaz, J. Sanchez Hubert, J. Sanchez Palencia, D. Vasiliev, On spectral pollution in the finite element approximation of thin elastic 'membrane' shells, Numer. Math., 75 (1997) 473-500.

[10] E. Shargorodsky, Geometry of higher order relative spectra and projection methods, J. Oper. Theo., 44 (2000) 43-62.

[11] Web page http://www.ma.hw.ac.uk/ lyonell/stable

(c) बEVIV, Zagreb

Paper No. 01-15 